\section{Who stands to lose from double-blind review?}

SIR - Why do journals expect scientists to use double-blind methods in their research, but refuse to apply a double-blind approach in evaluating that same research? The Editorial 'Working double-blind'(Nature 451, 605-606; 2008) addresses this long-standing puzzle, arguing in defence of the current system of single-blind review.

This echoes the attitude of most journals over the years. The reasons offered in support of this stance have gradually mutated with time - from the burden of removing author names, through the inefficiency of masking author identity, to the potential downsides of author anonymity. The underlying attitude has remained fundamentally unchanged.

This makes me wonder whether the invariable position of leading journals on double-blind review may in fact be the result of an invariable reason, the mention of which is consistently avoided. To venture a guess: could that reason be pressure from prominent members of the research community who are opposed to a system in which they cannot fully rely on the benefits of their reputation?

Boyan K. Garvalov

Max Planck Institute of Neurobiology,

Am Klopferspitz 18, 82152 Martinsried, Germany

\section{Double-blind review: the paw print is a giveaway}

SIR - In your Editorial 'Working doubleblind' (Nature 451, 605-606; 2008), you suggest that authors may be vulnerable to bias if referees guess their identities - for example, bias about their previous work, their gender, their nationality or their being new to a field. A high reputation could inhibit impartial assessment, although this bias is more likely to disarm than antagonize a reviewer.

The days when the gender or the nationality of a scientist could always be ascertained by her/his name are long gone; for example, try guessing my gender or nationality. I have met people from a nonEuropean background who thought that the French name Jean-Marie was a female name.

An author (or an entire team) embarking on a new area of research is easily spotted: the bibliography will contain no or few references to previous works by the author(s) of the paper under review. Scientists who frequently refer to their own work are readily identified; one does not have to be a Newton to be recognized by one's paw prints (Nature 333, 592; 1988). Double-blinding will not curb excessive self-citation because those who succumb to vanity know that they will be recognized anyway.
Regarding the open sharing of information, readers of a paper could benefit not just from the published paper, but also from the referees' reports if these were pithy and incisive.

Once upon a time, some journals appended to each paper the comments and the names of the reviewers. How about adopting this as an alternative to doubleblind review, and rewarding scholars for all their contributions, original as well as critical?

\section{K. Razi Naqvi}

Department of Physics, Norwegian University of Science and Technology, N-7491 Trondheim, Norway

\section{Double-blind review: easy to guess in specialist fields}

SIR - I work in a field, replicon dynamics in microbes, in which the cooperation-tocompetition ratio is, I would guess, relatively high. Subject, technique, citation habits and writing style would subvert more than $80 \%$ of attempts to conceal author identity, just as authors guess referee identity correctly in about $50 \%$ of cases . I doubt whether double-blinding, as discussed in your Editorial 'Working double-blind' (Nature 451, 605-606; 2008), would be worth doing in such a situation.

Is it really just at the journal-reviewer interface where reputation works most insidiously? One hears of big names stamping their foot over the telephone to intimidate editors into acceptance. Even if such tactics are occasionally tried, author-editor anonymity is too impracticable to consider as a corrective measure.

\section{David Lane}

Laboratoire de microbiologie et génétique moléculaires, CNRS, bât. IBCG, 118 route de Narbonne, 31062 Toulouse, France

\section{Double-blind review: let diversity reign}

SIR - The aim of blinding in peer-review is to improve the quality of reviews by removing any bias that might arise from knowing the identity of the authors. As your Editorial 'Working double-blind' (Nature 451, 605$606 ; 2008$ ) acknowledges, reviewing is not genuinely blind. You cite a study in which blinded reviewers identified at least one author on some $40 \%$ of papers (M. K. Cho et al. J. Am. Med. Assoc. 280, 243-245; 1998). The recognizable authors were presumably well-known, and anecdotally, these are the authors favoured by single-blinding. So the effect of double-blinding may be to increase the bias towards well-known researchers.
We need definitive data on this score. Meanwhile, journals are plentiful and varied and (although most use single blinding) they offer a choice from totally open to doubleblind reviewing. Switching to a different practice might help some journals to attract good manuscripts from scientists influenced by the type of review process.

Diversity is rated highly in ecology and economists lecture us about the benefits of free markets. Why not apply the same norms to the way we decide which papers to publish?

Bob O'Hara

Department of Mathematics and Statistics, PO Box 68 (Gustaf Hällströmin katu 2b), FIN-00014 University of Helsinki, Finland

Readers' comments on double-blinding are welcomed at http://tinyurl.com/2bzmvj.

\section{Tibet's seeds must be stored as climate changes}

SIR - The Tibet-Qinghai plateau is an area where climate change may have huge effects as glaciers retreat, leading to large decreases in water supply in the mega-rivers of India, southeast Asia and China by the middle of the century. For the 6,000 or more species of higher plants, including the widely admired Himalayan alpines, the effects will be even more severe as vegetation zones move upwards by several hundred metres. The movement of regions suitable for growth will be followed, not accompanied, by the vegetation suited to them, increasing the risk of extinctions.

In Tibet, few of the practices adopted in many other countries are in place. Although there are 38 nature reserves, covering a third of the country, there are no botanical gardens. The preservation of seeds of Tibetan plants is virtually non-existent. The Millennium Seed Bank at Kew in the United Kingdom stores seed from only three Tibetan species, and China's largest seed bank, the Southwest China Germplasm Bank of Wild Species in the Kunming Institute of Botany, has none.

We and researchers at other institutions are addressing this gap. We hope we'll be in time. W. John Cram ${ }^{\star}$, Yang Zhong ${ }^{\prime}$, Tashi Tersing;, Jie Cail|

${ }^{\star}$ China-UK HUST-RRes Genetic Engineering and Genomics Joint Laboratory, Huazhong University of Science and Technology, Wuhan, Hubei 430074, China

$\uparrow$ School of Life Sciences and Center for

Evolutionary Biology, Fudan University, Shanghai 200433, China

Institute of Biodiversity Science and Geobiology, Tibet University, Lhasa 850000, China

||Millennium Seed Bank Project, Royal Botanic Gardens, Kew, Wakehurst Place, Ardingly, West Sussex RH17 6TN, UK 